DE

M E D I C I N A

T R O P I C A L

$\mathrm{DE}$

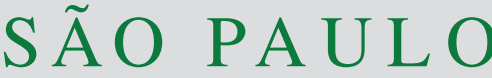

JOURNAL OF THE SÃO PAULO INSTITUTE OF TROPICAL MEDICINE

${ }^{1}$ Universidade Federal da Fronteira Sul, Chapecó, Santa Catarina, Brazil

${ }^{2}$ Instituto de Avaliação de Tecnologia em Saúde, Porto Alegre, Rio Grande do Sul, Brazil

${ }^{3}$ Hospital de Clínicas de Porto Alegre, Porto Alegre, Rio Grande do Sul, Brazil

${ }^{4}$ Universidade Federal do Rio Grande do Sul, Porto Alegre, Rio Grande do Sul, Brazil

${ }^{5}$ Hospital Moinhos de Vento, Porto Alegre, Rio Grande do Sul, Brazil

Correspondence to: Joanna d'Arc Lyra Batista

Universidade Federal da Fronteira Sul, Rodovia SC 459, Km 02, sala 317, Fronteira Sul, CEP 89801-001, Chapecó, SC, Brazil

E-mail: joanna.batista@uffs.edu.br

Received: 28 May 2020

Accepted: 26 October 2020

\section{A post-incorporation study on the use of palivizumab in the Brazilian public health system}

\author{
Joanna d'Arc Lyra Batista ${ }^{(1,2}$, Maria Angélica Pires Ferreira ${ }^{\left({ }^{2}, 3\right.}$, Cilene da \\ Silva Xavier ${ }^{\left({ }^{4}\right.}$, Ires Tarsila Alves de Souza ${ }^{(4}$, Luciane Nascimento Cruz ${ }^{(2,5}$, \\ Carisi Anne Polanczyk ${ }^{\mathbb{2}, 4,5}$
}

\section{ABSTRACT}

Respiratory syncytial virus (RSV) is the main cause of lower respiratory disease in infants and children under five years of age. As there is no specific treatment for RSV infections, prophylaxis with the specific monoclonal antibody palivizumab (PVZ) has been widely recommended for high-risk cases during the RSV season. The present study aimed to evaluate the effectiveness of a public prophylaxis program with palivizumab on the incidence of hospitalizations for lower respiratory tract infections and RSV in children at high risk for severe RSV infections. A retrospective cohort study was carried out with preterm children or children under two years of age with chronic lung disease or hemodynamically significant congenital heart disease; the children were selected on the basis of their exposure status, which was defined as the prophylactic use of palivizumab during the RSV season. Children were enrolled retrospectively in two hospitals located in Southern Brazil, from May 2009 to August 2016. In a sample of 129 children, $69(53.5 \%)$ received palivizumab and adherence to three or more doses was observed in 78\%; 60 (46.5\%) children did not receive palivizumab. PVZ prophylaxis was independently associated with a $66 \%$ reduction in hospitalizations for any cause (26/69 - 37.7\%) in the PVZ group and 34/60 (56.7\%) in the control group). A $52 \%$ reduction in hospitalizations due to lower respiratory tract infection was observed in the PVZ group (15/69 -21.7\%) and 25/60 (41.7\%) in the control group. These findings suggest that, for the group of studied patients, the adoption of an RSV prophylaxis scheme reached the same effectiveness as those described in previous clinical trials.

KEYWORDS: Palivizumab. Respiratory syncytial viruses. Infant health. Comparative effectiveness research.

\section{INTRODUCTION}

Respiratory syncytial virus (RSV) is one of the main etiological agents of infections affecting the lower respiratory tract among infants and children under five years of age, and it accounts for nearly $75 \%$ of bronchiolitis and $40 \%$ of pneumonia cases in infants up to one year of age during the RSV season ${ }^{1,2}$. Usually, the first infection progresses as an ordinary cold; however, severe RSV infections are responsible for hospitalization and, eventually, the need of mechanical ventilation (MV), mostly in children of less than 1 year of age ${ }^{1,3}$.

As there is no specific treatment for RSV infections, prophylaxis with the specific monoclonal antibody palivizumab (PVZ) has been widely recommended for high-risk cases during RSV season ${ }^{4}$. The groups with a higher risk of severe respiratory disease caused by RSV are premature infants, children with chronic 
lung disease of prematurity, immunosuppressed children and children suffering from heart conditions, especially those with hemodynamic consequences ${ }^{1}$.

The effectiveness of palivizumab in reducing hospitalizations due to RSV in infants has been demonstrated in randomized clinical trials, ranging from 39 to $78 \%$ in two pivotal clinical trials ${ }^{5-7}$. Evidence from observational studies suggests that PVZ prophylaxis reduces the incidence of recurrent wheezing over the first six years of life; however, it does not prevent the onset of atopic asthma ${ }^{8,9}$.

In December 2012, PVZ was introduced in the Brazilian public health system (SUS) for use in high-risk cases and premature infants at a gestational age equal to or less than 32 weeks $^{10}$; this decision was amended in 2013, with prophylaxis being recommended for infants under one year of age who were born prematurely at a gestational age equal to or less than 28 weeks, as well as in children up to two years of age suffering from chronic lung disease or hemodynamically significant congenital heart disease ${ }^{11}$.

Since the drug was incorporated into SUS, few studies have assessed the impact of the use of palivizumab on lower respiratory tract infection rates in children at high risk for respiratory syncytial virus infections. Post-incorporation studies are crucial to monitor the outcomes achieved with the use of this approach. The present study aimed to evaluate the effectiveness of a public prophylaxis program with palivizumab on the incidence of hospitalizations for lower respiratory tract infections and RSV cases in children at high-risk for severe RSV infections.

\section{MATERIAL AND METHODS}

\section{Study design}

A retrospective cohort study was conducted. The medical records of preterm children and/or children under two years of age suffering from chronic lung disease or hemodynamically significant congenital heart disease were reviewed for data collection. Children were selected according to their exposure status, which was defined as the prophylactic use of at least one dose of palivizumab with monthly doses of palivizumab during the RSV season (between April and August). Children were retrospectively recruited at the Hospital Materno Infantil Presidente Vargas (HMIPV) and Hospital de Clinicas de Porto Alegre RS (HCPA), both located in the city of Porto Alegre, in Southern Brazil. Although palivizumab was incorporated into the Brazilian public health system in 2013, due to the differences among Brazilian regions regarding drug acquisition and distribution and the issues of availability of electronic medical records in one of the centers, the study period started in 2014. The cases (PVZ group) were chosen from May 2014 to August 2016, a period subsequent to the incorporation of palivizumab into SUS, and controls were chosen from May 2009 to August 2016. The initial date for the controls recruitment was extended to include the period prior to $\mathrm{PVZ}$ incorporation due to the difficulty in gathering a sufficient number of children with indication but who did not take PVZ after 2014, in the health services assessed. The follow-up was censored in June 2017. The study period was up to two years following the first dose of palivizumab for exposed cases or up to two years after the identification of the indication criterium for the use of PVZ in the medical records, as recommended by the Ministry of Health for controls.

The study included children who met one of the following three criteria: 1) prematurity, defined as a gestational age at birth $\leq 28$ weeks; 2 ) less than two years of age with chronic lung disease resulting from prematurity; or 3) less than two years of age with hemodynamically significant congenital heart disease.

The list of children who received palivizumab was elaborated according to palivizumab dispensation records from the hospital pharmacies. Children in the control group were identified from the medical records of children hospitalized in neonatal or pediatric units during the study period, as indicated by International Classification of Diseases codes for prematurity or chronic lung disease resulting from prematurity or congenital heart disease. Children transferred from other health services were excluded.

Outcomes were assessed by analyzing data from the medical records according to the diagnosis made in the health service unit by the attending physician. The outcomes were grouped into four categories: 1) hospitalization for any reason (hospitalization rate); 2) hospitalization for lower respiratory tract infection regardless of etiology (ICD 10: J20 - J22); 3) hospitalization for respiratory syncytial virus infection (ICD 10: B97.4, J12.1, J20.5, or J21.0) and 4) death from any cause.

\section{Sample size and data analysis}

The sample size was calculated using the EPI-INFO software, version 7.2, considering the following parameters for a cohort study: $80 \%$ test power; $95 \%$ confidence level $(\alpha=5)$; an expected risk value to be detected of 0.6 ; and an expected emergency admission rate due to respiratory causes among the unexposed of $64 \%$ according to the pilot study conducted at the HCPA. The total sample size calculated based on these parameters was 134 (67 exposed and 67 unexposed). 
A descriptive analysis on the frequency distribution of risk factors was carried out according to the exposure groups in the cohort. Student's t test was used to compare the means for some characteristics between the PVZ and control groups.

As the cohort groups had different years of inclusion as it was difficult to find children with indications for the use that were not using prophylaxis after PVZ was incorporated into SUS, it was decided that the year of entry into the cohort would be included as an independent variable to be tested.

A logistic regression analysis was performed to identify predictors of hospitalization. The variables that were significant in the bivariate analysis (with a p-value $\leq 0.25$ ) were included in the multivariate logistic regression model. Backward and Irtest multivariate analyses were performed after the removal of each variable to verify whether this procedure had an impact on the model. The odds ratio (OR), relative risks (RR) and $95 \%$ confidence intervals were calculated. The data were entered into and stored in REDCap (Research Electronic Data Capture) and analyzed using STATA software, version 11.2 (Stata-Corp LP, College Station,Texas, USA). The study was approved by the ethics committees of Hospital Materno Infantil Presidente Vargas and Hospital de Clinicas de Porto Alegre.

\section{RESULTS}

One hundred and twenty-nine children were included in the study; $69(53.5 \%)$ were exposed to palivizumab (cases) and $60(46.5 \%)$ were in the unexposed group (controls). Most children (91/129 - 70.5) were included based on a gestational age equal to or less than 28 weeks and six days, followed by those with less than two years of age and with chronic lung disease of prematurity (28/129 - 21.7\%), less than two years of age and suffering from heart disease and severe lung hypertension (7/129 - 5.4\%), and those with more than two years of age suffering from complex congenital heart disease $(3 / 129-2.3 \%)$. Seventy nine children lived in the city of Porto Alegre (61.2\%), 106 were born from single pregnancies $(82.2 \%)$ and $91(70.5 \%)$ were breastfed. For the city of residence, there was a difference between the groups, as most of the unexposed (36/60 - 60\%) lived in other cities, while those who received PVZ (55/69 - 79.7\%) were mostly Porto Alegre residents $(\mathrm{p}<0.001)$. The control group children were less breastfed $(38 / 53-71.7 \%)$ than the group receiving palivizumab $(53 / 58-91.4 \%)(\mathrm{p}<0.01)$. Clinical and demographic characteristics are shown in Table 1.

Of the 69 children who received palivizumab, 28 (40.6\%) received five doses, $13(18.8 \%)$ received four doses, $13(18.8 \%)$ received three doses, $13(18.8 \%)$ received two doses, and two $(2.9 \%)$ received one dose. It was noted that, in some cases, the first dose was taken up to three months after the beginning of the RSV season. Among patients who received palivizumab, $46(66.7 \%)$ received all the necessary doses during the RSV season, 11 (15.9\%) missed only one month, and the remainder $12(17.4 \%)$ missed two months or more during the RSV season. Side effects were recorded in three $(4.3 \%)$ children: a case of apnea with cyanosis, a case of low fever after the administration of the first dose $\left(37.4{ }^{\circ} \mathrm{C}\right)$, and a case of cutaneous rash.

\section{Analysis of hospitalization for any cause}

Among children who did not receive palivizumab, 34 $(56.7 \%)$ were hospitalized during the follow-up, and among those who received palivizumab, 26 (37.7\%) children were hospitalized ( $\mathrm{p}=0.024)$. The mean hospital length of stay

Table 1 - Demographic and clinical characteristics of children meeting the criteria for the use of palivizumab, treated at Hospital de Clinicas de Porto Alegre and Hospital Presidente Vargas, Porto Alegre, 2017.

\begin{tabular}{lccc}
\hline Characteristics & $\begin{array}{c}\text { Using PVZ } \\
\text { mean }\end{array}$ & $\begin{array}{c}\text { Not using PVZ } \\
\text { mean }\end{array}$ & $P^{*}$ \\
\hline $\begin{array}{l}\text { Age at PVZ indication } \\
\text { months }\end{array}$ & 7.33 & 3.67 & $\mathbf{0 . 0 0 0}$ \\
$\begin{array}{l}\text { Weight at birth } \\
\quad \text { grams }\end{array}$ & 1.177 & 1.059 & $\mathbf{0 . 1 8 8}$ \\
$\begin{array}{l}\text { Gestational age at birth } \\
\quad \text { weeks }\end{array}$ & 29.24 & 29.14 & $\mathbf{0 . 8 5 5}$ \\
$\begin{array}{l}\text { Number of people in household } \\
\text { Length of stay in the neonatal ICU } \\
\text { days }\end{array}$ & 4.39 & 4.71 & $\mathbf{0 . 4 0 1}$ \\
\hline
\end{tabular}

${ }^{\star} P$ from Student's t test 
in the palivizumab group was 9.54 days, and in the control group, it was 8.75 days $(\mathrm{p}=0.81)$. Among hospitalized patients, only five children needed mechanical ventilation, three from the control group and two from the PVZ group. The mean mechanical ventilation time was 6.3 days for the controls and 3.0 days for those who received palivizumab $(\mathrm{p}=0.12)$.

The main reason for hospitalization was bronchopneumonia, which was responsible for $23.3 \%$ of the total hospitalizations (14/60), followed by acute bronchiolitis $(10 / 60-16.7 \%)$, acute bronchitis $(6 / 60-10 \%)$, acute bronchiolitis caused by RSV (6/60 or 10\%), and asthma $(5 / 60-8.3 \%)$. All other reasons for hospitalization accounted for only one case (1/60 - $1.7 \%)$ each.

The incidence rate of hospitalization for any cause was 45.7 per 100 people/year (95\% CI: 35.5-58.9). In the group of children who did not take palivizumab (control group), the density of hospitalization incidence was 59.2 per 100 people/year (95\% CI: 42.3-82.9), and in the children who used palivizumab, it was 35.2 per 100 people/year (95\% CI: 23.9-51.7).

Table 2 shows the bivariate analyses of variables associated with hospitalization for any cause. There was no association between the year of entry into the cohort and hospitalization for any cause, indicated by a p-value $>0.25$ $(\mathrm{p}=0.879)$; therefore, this variable was not included in the multivariate model (data not shown in the table).

Table 3 shows the final result of the multivariate model for predictors of hospitalization for any cause. The variables with $\mathrm{p} \leq 0.25$ were added to the saturated model and then removed one by one from the model as the p-value increased, until only those that were statistically significant $(\mathrm{p}<0.05)$ remained. Only the use of palivizumab remained in the final model as a significant variable.

There was no difference in the number of doses of PVZ between children hospitalized for any reason (mean of 3.73 doses) and those that were not hospitalized (mean of 3.77 doses; $\mathrm{p}=0.91$ ).

Table 2 - Bivariate analysis of parameters associated with hospitalization for any cause in children with indication for the use of palivizumab, Porto Alegre, 2017.

\begin{tabular}{|c|c|c|c|c|}
\hline & \multicolumn{2}{|c|}{ Hospitalization for any cause } & \multirow[b]{2}{*}{ OR (95\% Cl) } & \multirow[b]{2}{*}{$p$} \\
\hline & $\begin{array}{c}\text { Yes } \\
\mathrm{n}(\%)\end{array}$ & $\begin{array}{c}\text { No } \\
n(\%)\end{array}$ & & \\
\hline \multicolumn{5}{|c|}{ Use of prophylactic palivizumab } \\
\hline Yes & $26(37.7)$ & $43(62.3)$ & 1.0 & \\
\hline No & $34(56.7)$ & $26(43.3)$ & $0.46(0.23-0.94)$ & 0.032 \\
\hline \multicolumn{5}{|l|}{ Sex } \\
\hline Female & $32(45.7)$ & $38(54.3)$ & 1.0 & \\
\hline Male & $28(47.5)$ & $31(52.5)$ & $1.07(0.54-2.15)$ & 0.843 \\
\hline \multicolumn{5}{|l|}{ Place of residence } \\
\hline Porto Alegre & $35(44.3)$ & $44(55.7)$ & 1.0 & \\
\hline Another city & $25(50.0)$ & $25(50.0)$ & $1.26(0.62-2.56)$ & 0.528 \\
\hline \multicolumn{5}{|c|}{ Number of people in household } \\
\hline Up to 3 people & $09(37.5)$ & $15(62.5)$ & 1.0 & \\
\hline 4 people or more & $22(37.9)$ & $36(62.1)$ & $1.02(0.38-2.72)$ & 0.971 \\
\hline \multicolumn{5}{|c|}{ Main reason for inclusion in the study } \\
\hline Prematurity & $46(50.5)$ & $45(49.5)$ & 1.0 & \\
\hline Lung or heart disease & $14(36.8)$ & $24(63.2)$ & $0.57(0.26-1.24)$ & 0.157 \\
\hline \multicolumn{5}{|l|}{ Breastfeeding records } \\
\hline Yes & $43(47.2)$ & $48(52.8)$ & 1.0 & \\
\hline No & $09(45.0)$ & $11(55.0)$ & $0.91(0.34-2.41)$ & 0.855 \\
\hline \multicolumn{5}{|l|}{ For those who used PVZ: } \\
\hline \multicolumn{5}{|c|}{ Number of doses administered } \\
\hline Three or more & $21(38.9)$ & $33(61.1)$ & 1.0 & \\
\hline Up to two & 05 (33.3) & $10(66.7)$ & $0.78(0.23-2.62)$ & 0.695 \\
\hline
\end{tabular}

${ }^{*}$ Adjusted per year of entry into the study 
Table 3 - Final multivariate analysis of the association between hospitalization for any cause and exposure elements of individuals, Porto Alegre, 2017.

\begin{tabular}{|c|c|c|c|c|c|}
\hline & $\begin{array}{c}\text { Odds Ratio and } \\
95 \% \mathrm{Cl}\end{array}$ & $p$ & $\begin{array}{l}\text { Relative Risk and } \\
95 \% \mathrm{Cl}\end{array}$ & $p$ & PAF \\
\hline \multicolumn{6}{|c|}{ Use of prophylactic palivizumab } \\
\hline Yes & 1.0 & & 1.0 & & \\
\hline No & $0.46(0.23-0.94)$ & 0.032 & $0.66(0.46-0.97)$ & 0.031 & -0.50 \\
\hline
\end{tabular}

Analysis of hospitalization due to lower respiratory tract infections

Among the children using palivizumab, hospitalizations for lower respiratory tract infections were noted in $21.7 \%$ $(15 / 69)$, while in those who did not receive palivizumab, hospitalizations were observed in $41.7 \%(25 / 60)(\mathrm{p}=0.01)$. The incidence rate of hospitalization for lower respiratory tract infection was 26.0 per 100 people/year (95\% CI: 19.1 - 35.5). In children who did not take palivizumab (control group), the density of hospitalization incidence was 35.3 per 100 people/year (95\% CI: 23.9 - 52.2), and in the children who used palivizumab, the calculated rate was 18.1 per 100 people/year (95\% CI: 10.9 - 30.0).

Table 4 shows the bivariate analyses of elements possibly associated with hospitalization for lower respiratory tract

Table 4 - Bivariate analysis of parameters associated with hospitalization for lower respiratory tract infections in children with indication for the use of palivizumab, Porto Alegre, 2017.

\begin{tabular}{|c|c|c|c|c|}
\hline & \multicolumn{2}{|c|}{$\begin{array}{c}\text { Hospitalization for lower respiratory tract } \\
\text { infections }\end{array}$} & \multirow{2}{*}{ OR $(95 \% \mathrm{Cl})$} & \multirow{2}{*}{$p$} \\
\hline & $\begin{array}{l}\text { Yes } \\
\mathrm{n}(\%)\end{array}$ & $\begin{array}{c}\text { No } \\
n(\%)\end{array}$ & & \\
\hline \multicolumn{5}{|c|}{ Use of prophylactic palivizumab } \\
\hline Yes & $15(21.7)$ & $54(78.3)$ & 1.0 & \\
\hline No & $25(41.7)$ & $35(58.3)$ & $0.39(0.18-0.84)$ & 0.016 \\
\hline \multicolumn{5}{|l|}{ Sex } \\
\hline Female & $24(34.3)$ & $46(65.7)$ & 1.0 & \\
\hline Male & $16(27.1)$ & $43(72.9)$ & $0.71(0.33-1.52)$ & 0.381 \\
\hline \multicolumn{5}{|l|}{ Place of residence } \\
\hline Porto Alegre & $21(26.6)$ & $58(73.4)$ & 1.0 & \\
\hline Another city & $19(38.0)$ & $31(62.0)$ & $1.69(0.79-3.61)$ & 0.174 \\
\hline \multicolumn{5}{|c|}{ Number of people in household } \\
\hline Up to 3 people & $04(16.7)$ & $20(83.3)$ & 1.0 & \\
\hline 4 people or more & $16(27.6)$ & $42(72.4)$ & $1.90(0.56-6.44)$ & 0.300 \\
\hline \multicolumn{5}{|c|}{ Main reason for inclusion in the study } \\
\hline Prematurity & $31(34.1)$ & $60(65.9)$ & 1.0 & \\
\hline Lung or heart disease & $09(23.7)$ & $29(76.3)$ & $0.60(0.25-1.42)$ & 0.248 \\
\hline \multicolumn{5}{|l|}{ Breastfeeding records } \\
\hline Yes & $29(31.9)$ & $62(68.1)$ & 1.0 & \\
\hline No & $07(35.0)$ & $13(65.0)$ & $1.15(0.41-3.19)$ & 0.787 \\
\hline \multicolumn{5}{|l|}{ For those who used PVZ: } \\
\hline \multicolumn{5}{|c|}{ Number of doses administered } \\
\hline Three or more & $11(20.4)$ & $43(79.6)$ & 1.0 & \\
\hline Up to two & $04(26.7)$ & $11(73.3)$ & $1.42(0.38-5.33)$ & 0.602 \\
\hline
\end{tabular}

${ }^{*}$ Adjusted per year of entry into the study 
Table 5 - Final multivariate analysis of the association between hospitalization for lower respiratory tract infections and exposure elements of individuals, Porto Alegre, 2017.

\begin{tabular}{lccccc}
\hline & $\begin{array}{c}\text { Odds Ratio and } \\
95 \% \mathrm{Cl}\end{array}$ & $p$ & $\begin{array}{c}\text { Relative Risk and } \\
95 \% \mathrm{Cl}\end{array}$ & $p$ & PAF \\
\hline $\begin{array}{l}\text { Use of prophylactic palivizumab } \\
\text { Yes }\end{array}$ & $\begin{array}{c}1.0 \\
\text { No }\end{array}$ & & 1.0 & & \\
No & $0.39(0.18-0.84)$ & 0.016 & $0.52(0.30-0.89)$ & 0.014 & -0.59 \\
\hline
\end{tabular}

infections. The bivariate analysis of the association between the year of entry into the cohort and hospitalization for lower respiratory tract infections showed a p value $>0.25$ $(\mathrm{p}=0.43)$; thus, it was not a candidate for inclusion in the multivariate model (data not shown in the table).

Table 5 shows the results of the final multivariate analysis model for the association between the studied parameters and hospitalization for lower respiratory tract infections. Variables were removed from the model as the p-value increased until only the statistically significant ones remained $(\mathrm{p}<0.05)$.

Six hospitalizations with laboratory confirmation of respiratory syncytial virus infections were recorded, all of which were in the control group. The rate of respiratory syncytial virus infection in the cohort was 3.2 per 100 people/year (95\% CI: $1.42-7.04)$. As this was a retrospective study, RSV infection was only considered when the diagnosis was recorded in the medical records, and it is not possible to know whether all the patients were actually tested for RSV, as this is a retrospective study.

\section{General analysis of the sample}

The use of palivizumab remained independently associated with hospitalization for any cause and hospitalization for lower respiratory tract infections, was a protective factor, with relative risk reductions of $66 \%$ and $52 \%$, respectively. The population attributable fraction associated with the use of palivizumab for hospitalizations for any cause was -0.50 and for hospitalization for lower respiratory tract infections was -0.59 ; that is, it is estimated that palivizumab decreases hospitalizations for any cause by $50 \%$ and hospitalizations for lower respiratory tract infections by $59 \%$.

Only one death was observed in the cohort, and it occurred 1.2 years after the date of inclusion; this child was in the control group. The death rate in the cohort was 3.7 per 1,000 people/year (95\% CI: 0.53-26.6).

\section{DISCUSSION}

Palivizumab prophylaxis was independently associated with a reduction in hospitalizations for any cause and hospitalization due to lower respiratory tract infections in patients at high risk of RSV infection.

The two-year rate of hospitalization for any cause in the cohort was $46.5 \%$ and it is noteworthy that this was a more vulnerable group, given that children were premature and/ or suffered from pulmonary or cardiacdiseases. The hospitalization rate among those who did not receive palivizumab was higher for both, hospitalizations for any cause $(56.7 \%)$ and hospitalizations for lower respiratory tract infections (41.7\% versus $31 \%$ ). These findings are corroborated by the result of the multivariate analyses as well as the calculation of the population attributable fraction, according to which, palivizumab reduced hospitalizations for any cause by $50 \%$ and hospitalizations for lower respiratory tract infections by $59 \%$.

The rate of hospitalization for lower respiratory tract infections among those who received palivizumab (21.7\%) was higher than the rate of hospitalization for acute respiratory tract infections (12.9\%) described in Canada during the 2004 and 2005 seasons $^{12}$. It is important to stress that regarding the children who received palivizumab that we assessed, the first dose was administered up to three months after the beginning of the RSV season, which may indicate a delay in palivizumab prescription or in the patient's visit to the referral center after receiving the palivizumab prescription at the original hospital.

The effectiveness of palivizumab in reducing hospitalization due to respiratory syncytial virus was shown in the IMpact-RSV study ${ }^{6}$, a randomized double-blind clinical trial that included 1,502 premature children (up to 35 weeks of gestational age) or with bronchopulmonary dysplasia; the trial was conducted in the United States, United Kingdom and Canada in the late 1990s. In that trial, a 55\% decrease in hospitalizations for RSV was observed in the group treated with palivizumab (4.8\%) compared with the group that received placebo $(10.6 \%)$. The hospitalization rate for RSV in the placebo group of the IMpact-RSV study was similar to the rate found in those unexposed to PVZ in our study, which was $10 \%$. In another multicenter clinical trial $^{5}$, a relative decrease of $45 \%$ in hospitalizations for RSV resulting from the use of palivizumab was described. A systematic review carried out in 2011 has also evaluated the 
RSV hospitalization rate among groups who received PVZ, the summarized measure of which was 0.35 for the premature infants group $(\mathrm{p}<0.001)^{13}$. Although it was not possible to assess the reduction in RSV-specific hospitalizations in this study as this outcome did not occur in the exposed group, the observed data indicate that the use of palivizumab decreases hospitalizations for any cause by $50 \%$.

The clinical trials comparing exposure to palivizumab with the use of placebo in the literature are international studies with different realities in terms of respiratory infection profiles. The studies that provided the basis for indicating the incorporation of palivizumab by CONITEC were published prior to 2010, and little has been done after this incorporation, especially in view of the ethical issue that makes it difficult to gather/ form a control group. In Brazil, studies on the topic are scarce, and our results can only be compared with the cohort study conducted by Monteiro et al. ${ }^{1}$, in 2014 with patients subjected to palivizumab prophylaxis; this study was performed without a control group for ethical reasons. Among the children who received palivizumab, the rate of hospitalization for lower respiratory tract infections was $9.1 \%{ }^{1}$, a much lower rate than that found in this study (21.7\%). The Southern region of the country may present a different respiratory infection profile due to climate characteristics, with a more distinct winter and lower temperatures, as well as to an increase in the seasonality of RSV and other respiratory viruses.

As this was a retrospective study, it was not possible to ensure that all patients with acute lower respiratory tract infections underwent laboratory tests for respiratory syncytial virus, which may explain the low rate of RSV-positive infections among the patients with bronchiolitis, bronchitis and bronchopneumonia (16.7\%). This percentage is much lower than the rate found by Souza in Porto Alegre ${ }^{14}$, who identified RSV in 59\% of samples from infants up to 12 months of age with bronchiolitis; unfortunately, these patients were recruited between September 2009 and August 2011, prior to the incorporation of PVZ by the public health system.

There was a difference in the mean age of the patients chosen for the cohort, with the group using palivizumab being in general older than the unexposed group. This difference may have resulted from the fact that the controls were included upon the observation of the indication of palivizumab in accordance with criteria specified by the Brazilian Ministry of Health, while the cases that received palivizumab were included from the date of the first dose, which indicates the date of initial exposure to the drug under study. As there is a difference between the date of prescription and the first date the drug was administered, especially because the only referral hospital for the treatment with palivizumab was the HMIPV, there may have been a delay in the patient's visit to this specialized service. At the HCPA, the doses were administered only to hospitalized patients, while outpatients were referred to the HMIPV.

Another difference found between the exposure groups was related to the patients' cities of residence. Most children in the group unexposed to palivizumab $(60 \%)$ lived in cities other than the capital city Porto Alegre.

The hospitals included in this study are referral hospitals located in the metropolitan area of Porto Alegre. The unexposed patients from other cities may have risen questions about the prescription and the use of palivizumab in hospitals located in their home cities; however, to avoid this bias, only patients with at least two years of outpatient follow-up at the referral hospital and frequent visits were selected to ensure that their medical records really included information on medications occasionally administered in their cities of residence.

An important point in the monitoring of new components incorporated into the public health system should take into account the cost of prophylaxis in relation to the benefits it offers, ideally through a complete cost-effectiveness study. It would be important to analyze the increase in drug costs to the budget of the public health system, taking into account favorable outcomes, such as a decrease in hospitalizations and problems for patients and their families. Unfortunately, these economic issues were not analyzed in this study and should be addressed in future research.

The limitations of the study were those inherent to the retrospective cohort study design itself, such as the inability to retrospectively collect some variables that are possibly association confounders (in this case, socioeconomic variables). Regarding the external validity, this study produced specific results as it was conducted at referral hospitals and in a capital city located in the Southern region of the country, where seasonality is well defined and high incidence levels of respiratory infections are often experienced in winter, what makes this region stand out from the rest.

\section{CONCLUSION}

The use of PVZ has been approved in several countries, although its effectiveness is controversial in developing countries and in places with a low incidence of respiratory infections. In Brazil, there were many questions regarding the effectiveness of this drug for the Brazilian population at the time of its recommendation in view of sociodemographic, epidemiological and even geographical differences. This study sought to address a key aspect of monitoring the incorporation of new concepts into the public health system in an attempt to reinvigorate the process 
based on the successes and mistakes resulting from the decisions made. The results suggest that the adoption of this prophylactic program achieved the expected effectiveness for the studied patients corroborating the findings of previously published international clinical trials.

\section{ACKNOWLEDGMENTS}

We thank Dr. Ana Maria Araujo Cirne and Dr. Cintia Beatriz Momo Selister for their support in data collection.

\section{AUTHORS' CONTRIBUTIONS}

JDLB and CAP participated in the study design; data collection, analysis and interpretation; and writing of the manuscript. MAPF, CSX and ITAS participated in the data collection; interpretation of the findings; and writing of the manuscript. LNC participated in the interpretation of the findings; and writing of the manuscript. All authors read and approved the final version of the manuscript.

\section{CONFLICT OF INTERESTS}

The authors declare no conflict of interests.

\section{FUNDING}

The study was sponsored and coordinated by the Moinhos de Vento Hospital, Brazil, in partnership with the Brazilian Ministry of Health through the Program of Institutional Development of the Brazilian Unified Health System (PROADI-SUS) (Project 01553 - ATS/PROADI HMV).

\section{REFERENCES}

1. Monteiro AI, Bellei NC, Sousa AR, Santos AM, Weckx LY. Respiratory infections in children up to two years of age on prophylaxis with palivizumab. Rev Paul Pediatr. 2014;32:152-8.

2. Monteiro CC, Dezanete LN, França EB. Monitoramento de vírus respiratórios na região metropolitana de Belo Horizonte, 2011 a 2013. Epidemiol Serv Saude. 2016;25:233-42.

3. Kenmoe S, Kengne-Nde C, Modiyinji AF, La Rosa G, Njouom R. Comparison of health care resource utilization among preterm and term infants hospitalized with Human Respiratory Syncytial Virus infections: a systematic review and meta-analysis of retrospective cohort studies. PLoS One. 2020;15:e0229357.

4. Sáfadi MA. Immunoprophylaxis against respiratory syncytial virus with palvizumab: what is new? Rev Paul Pediatr. 2014;32:150-1.
5. Feltes TF, Cabalka AK, Meissner HC, Piazza FM, Carlin DA, Top Jr FH, et al. Palivizumab prophylaxis reduces hospitalization due to respiratory syncytial virus in young children with hemodynamically significant congenital heart disease. J Pediatr. 2003;143:532-40.

6. Palivizumab, a humanized respiratory syncytial virus monoclonal antibody, reduces hospitalization from respiratory syncytial virus infection in high-risk infants. The IMpact-RSV Study Group. Pediatrics. 1998;102:531-7.

7. Andabaka T, Nickerson JW, Rojas-Reyes MX, Rueda JD, Vrca VB, Barsic B. Monoclonal antibody for reducing the risk of respiratory syncytial virus infection in children. Cochrane Database Syst Rev. 2013;CD006602.

8. Yoshihara S, Kusuda S, Mochizuki H, Okada K, Nishima $\mathrm{S}$, Simões EA. Effect of palivizumab prophylaxis on subsequent recurrent wheezing in preterm infants. Pediatrics. 2013;132:811-8.

9. Mochizuki H, Kusuda S, Okada K, Yoshihara S, Furuya H, Simões EA. Palivizumab prophylaxis in preterm infants and subsequent recurrent wheezing: six-year follow-up study. Am J Respir Crit Care Med. 2017;196:29-38.

10. Brasil. Ministério da Saúde. Secretaria de Ciência, Tecnologia e Insumos Estratégicos. Departamento de Gestão e Incorporação de Tecnologias em Saúde. Palivizumabe para a prevenção da infecção pelo vírus sincicial respiratório: dezembro de 2012: relatório de recomendação da Comissão Nacional de Incorporação de Tecnologias no SUS-CONITEC-16. Brasília: Ministério da Saúde; 2012. [cited 2020 Oct 26]. Available from: http://conitec.gov.br/images/Incorporados/PalivizumabeVirusSincicial-final.pdf

11. Brasil. Ministério da Saúde. Gabinete do Ministro. Portaria $\mathrm{n}^{\circ} 522$, de 13 de maio de 2013. Aprova o protocolo de uso do Palivizumabe. Diário Oficial da União, Brasília, 15 maio 2013. Seção 1:43-5. [cited 2020 Oct 26]. Available from: http://bvsms.saude.gov.br/bvs/saudelegis/sas/2013/ prt0522_13_05_2013.html

12. Boivin G, Caouette G, Frenette L, Carbonneau J, Ouakki M, De Serres G. Human respiratory syncytial virus and other viral infections in infants receiving palivizumab. J Clin Virol. 2008;42:52-7.

13. Checchia PA, Nalysnyk L, Fernandes AW, Mahadevia PJ, Xu Y, Fahrbach K, et al. Mortality and morbidity among infants at high risk for severe respiratory syncytial virus infection receiving prophylaxis with palivizumab: a systematic literature review and meta-analysis. Pediatr Crit Care Med. 2011;12:580-8.

14. Souza AP, Leitão LA, Luisi F, Souza RG, Coutinho SE, Silva JR, et al. Falta de associação entre carga viral e gravidade da bronquiolite aguda em lactentes. J Bras Pneumol. 2016;42:261-5. 